\title{
Chance-constrained games with mixture distributions
}

\author{
Shen Peng • Navnit Yadav • Abdel \\ Lisser • Vikas Vikram Singh
}

Received: date / Accepted: date

\begin{abstract}
In this paper, we consider an $n$-player non-cooperative game where the random payoff function of each player is defined by its expected value and her strategy set is defined by a joint chance constraint. The random constraint vectors are independent. We consider the case when the probability distribution of each random constraint vector belongs to a subset of elliptical distributions as well as the case when it is a finite mixture of the probability distributions from the subset. We propose a convex reformulation for the joint chance constraint of each player and derive the bounds for players' confidence levels and the weights used in the mixture distributions. Under mild conditions on the players' payoff functions, we show that there exists a Nash equilibrium of the game when the players' confidence levels and the weights used in the mixture distributions are within the derived bounds. As an application of these games, we consider the competition between two investment firms on the same set of portfolios. We use a best response algorithm to compute a Nash equilibrium of the randomly generated games of different sizes.
\end{abstract}

Shen Peng

Optimization and Systems Theory, Department of Mathematics, KTH Royal Institute of Technology, SE-100 44 Stockholm, Sweden

E-mail: shenp@kth.se

Navnit Yadav

Department of Mathematics, Indian Institute of Technology Delhi, Hauz Khas, New Delhi, 110016, India.

E-mail: navnitydv@gmail.com

Abdel Lisser

L2S, CentraleSupélec Bât Breguet A4.22 3 rue Joliot Curie 91190 Gif-sur-Yvette, France

E-mail: abdel.lisser@12s.centralesupelec.fr

Vikas Vikram Singh

Department of Mathematics, Indian Institute of Technology Delhi, Hauz Khas, New Delhi, 110016, India.

E-mail: vikassingh@iitd.ac.in 
Keywords Chance-constrained game - Mixture of elliptical distributions • Nash equilibrium · Portfolio.

\section{Introduction}

In strategic games, the rational players choose their strategies simultaneously which results in payoffs for all the players. The payoffs of each player depend on the strategies of all the players whereas the strategy set of each player does not depend on other players' strategies. The Nash equilibrium concept is used to analyze the outcome of such strategic interactions. Nash [23] showed that there exists a mixed strategy Nash equilibrium of a game with finite number of players where each player has finite number of actions. Since then, general strategic games have been extensively studied in the literature [1, 10, 11. The games considered in the above-mentioned papers are deterministic in nature, i.e., the payoffs and the strategy sets of all the players are defined by real valued functions. However, the decision making process is often subject to uncertainties due to the presence of various external factors [9, 22. The stochastic modelling of uncertainties present in the system depends on the attitude of decision makers. The expected value criterion is used for risk neutral players 25. For risk averse players, the payoff criterion with the risk measure CVaR [18, 25 and the variance are considered in the literature 8. A chance constraint based payoff criterion for the case of risk averse players is introduced in [26, 27. Singh et al. [26] considered a finite strategic games where the payoff vector of each player is a random vector, and each player is interested in payoffs obtained with a given confidence level. They showed that there exists a mixed strategy Nash equilibrium when the payoff vector of each player follows a multivariate elliptical distribution. In [27, the authors showed that the Nash equilibria of chance-constrained games (CCGs) considered in [26] can be obtained from the global optimal solutions of a certain mathematical program. Notice that there are zero-sum CCGs studied in the literature, see for instance [2, 4, 5, 6].

The above-mentioned papers on CCGs considered the case where players' payoffs are random and the strategy sets are deterministic in nature. There is a scarce literature on games with stochastic strategy sets defined by chance constraints [24, 28]. Singh and Lisser [28, showed that a saddle point equilibrium of a zero-sum game with individual chance constraints can be obtained by solving a primal-dual pair of second order cone programs. Peng et al. 24] considered $n$-player games with deterministic payoff functions and the strategy sets defined using joint chance constraints. They showed that there exists a Nash equilibrium of the game when the random constraint vectors are independent, and follow a multivariate normal distribution. A normal distribution belongs to the class of elliptical distributions. In practical situations, a normal distribution is often used to model the uncertain parameters, but, sometimes the member distributions from elliptical class other than normal distribution are used to model the uncertainties present in the system. For example, in fi- 
nancial market the random return vector from a portfolio follows an elliptical distribution [16] as well as a finite mixture of elliptical distributions [3, 13, 17].

In this paper, we consider an n-player game where the payoff function of each player is random and the strategy set of each player is defined by a joint chance constraint. The players' payoff functions are defined using expected value of their random payoff functions and the random constraint vectors are independent. We identify a list of prominent probability distributions belonging to the class of elliptical distributions. Under mild conditions, we show that there exists a Nash equilibrium of these CCGs if the underlying probability distributions of random constraint vectors belong to the list. Then, we consider the case where the random constraint vectors follow a finite mixture of elliptical distributions from the list and we show that there exists a Nash equilibrium of the CCG. The CCGs considered in this paper are significantly different from the ones considered in [24]. Unlike [24], we consider expected payoff functions for each player and the existence of Nash equilibrium is shown for certain elliptical distributions and their finite mixture. As an application of the CCG under mixture distributions, we consider the case of two investment firms which compete on the same market by investing in the same set of portfolios. The random return vector of each firm follows a finite mixture of elliptical distributions. The firms want to keep their random losses below a certain threshold. The constraints on the random loss function of each player are modelled as a joint chance constraint. We consider the case where each firm incurs transaction cost which depends on the strategies of both the firms. Each firm is interested in maximizing its expected payoffs over the chanceconstrained strategy set. We use a best response algorithm to compute the Nash equilibrium of the game. The numerical experiments are performed on randomly generated instances of different sizes.

The rest of the paper is organized as follows. Section 2 contains the definition of the CCGs. In Sections 3 and 4 . we show the existence of a Nash equilibrium for a subset of elliptical distributions, and for a finite mixture of elliptical distributions from the subset, respectively. Section 5 contains the application of the CCG in financial market. We conclude our paper in Section 6.

\section{The model}

We consider an $n$-player non-cooperative game, where $I=\{1,2, \ldots, n\}$ denotes the set of players. Let $X^{i} \subset \mathbb{R}_{++}^{l_{i}}$ be a strategy set of player $i$ which is a convex and compact set; $\mathbb{R}_{++}^{l_{i}}$ denotes the positive orthant of $\mathbb{R}^{l_{i}}$. The generic element of $X^{i}$ is denoted by $x^{i}$ and the vector of strategies of all the players except player $i$ is denoted by $x^{-i}$. The strategy profile of all the players is denoted by $x=\left(x^{i}, x^{-i}\right)$. Let $\xi: \Omega \rightarrow \mathbb{R}^{d}$ be a random vector defined on probability space $(\Omega, \mathcal{F}, \mathbb{P})$. For each $\omega \in \Omega$, let $v_{i}(x, \xi(\omega))$ represents a real valued payoff function of player $i$ which is defined on $\prod_{i \in I} \mathbb{R}_{++}^{l_{i}} \times \mathbb{R}^{d}$. The players use 
expected payoff criterion and the payoff function of player $i$ is defined as

$$
u_{i}(x)=\mathbb{E}_{\mathbb{P}}\left[v_{i}(x, \xi)\right],
$$

where $\mathbb{E}_{\mathbb{P}}$ denotes the expectation operator associated with probability measure $\mathbb{P}$. The strategy set of player $i, i \in I$, is restricted by the following joint chance constraint

$$
\mathbb{P}\left\{A^{i} x^{i} \leq b^{i}\right\} \geq \alpha_{i},
$$

where $\alpha_{i}$ is confidence level of player $i, A^{i}=\left[A_{1}^{i}, A_{2}^{i}, \ldots, A_{K_{i}}^{i}\right]^{T}$ is a $K_{i} \times l_{i}$ random matrix defined on the probability space $(\Omega, \mathcal{F}, \mathbb{P})$, and $b^{i} \in \mathbb{R}^{K_{i}} ; T$ denotes the transposition. For each $k=1,2, \ldots, K_{i}, A_{k}^{i}$ is the $k^{\text {th }}$ row of $A^{i}$. For a given $\alpha_{i}$, the feasible strategy set of player $i$ is defined by

$$
S_{\alpha_{i}}^{i}=\left\{x^{i} \in \mathbb{R}_{++}^{l_{i}} \mid x^{i} \in X^{i}, \mathbb{P}\left\{A^{i} x^{i} \leq b^{i}\right\} \geq \alpha_{i}\right\}, \forall i \in I .
$$

Let $\alpha=\left(\alpha_{i}\right)_{i \in I}$ be a confidence level vector. We denote $S_{\alpha}=\prod_{i \in I} S_{\alpha_{i}}^{i}$ and $S_{\alpha_{-i}}^{-i}=\prod_{j \in I ; j \neq i} S_{\alpha_{j}}^{j}$. We assume that the set $S_{\alpha_{i}}^{i}$ is nonempty, and the probability distribution of the random matrix $A^{i}$ and the probability level vector $\alpha$, and the payoff functions defined by (1) are known to all the players. We call this game a CCG which is a non-cooperative game with complete information. A strategy profile $x^{*}=\left(x^{i *}, x^{-i *}\right) \in S_{\alpha}$ is a Nash equilibrium of the CCG at confidence level vector $\alpha$ if and only if for each $i \in I$,

$$
u_{i}\left(x^{i *}, x^{-i *}\right) \geq u_{i}\left(x^{i}, x^{-i *}\right), \forall x^{i} \in S_{\alpha_{i}}^{i}
$$

Assumption 1 For each player $i, i \in I$, the following conditions hold.

1. $v_{i}\left(x^{i}, x^{-i}, \xi\right)$ is a concave function of $x^{i}$ for every $\left(x^{-i}, \xi\right) \in X^{-i} \times \mathbb{R}^{d}$.

2. $v_{i}(\cdot)$ is a continuous function.

3. $\mathbb{E}_{\mathbb{P}}\left[v_{i}(x, \xi)\right]$ is finite valued for any $x \in X$.

In this paper, we consider the case where the row vectors $A_{k}^{i}, k=$ $1,2, \ldots, K_{i}$, of matrix $A^{i}, i \in I$, are independent [15]. We select a subset of elliptical distributions given by Table 1, and we show that there exists a Nash equilibrium of CCG. In addition, we show that a Nash equilibrium exists when the probability distribution is a finite mixture of the elliptical distributions listed in Table 1.

Definition 1 A $d$-dimensional random vector $\zeta$ follows an elliptically symmetric distribution $\operatorname{Ellip}_{d}(\mu, \Sigma, \varphi)$ with location parameter $\mu$, positive definite scale matrix $\Sigma$, and characteristic generator $\varphi$, if its characteristic function is given by $\mathbb{E} e^{i t^{T} \zeta}=e^{i t^{T} \mu} \varphi\left(t^{T} \Sigma t\right)$. 
The probability density function (if it exists) of an elliptically symmetric distribution is given by

$$
f(\mathbf{u})=\frac{c}{\sqrt{\operatorname{det} \Sigma}} g\left(\sqrt{(\mathbf{u}-\mu)^{T} \Sigma^{-1}(\mathbf{u}-\mu)}\right),
$$

where $g(\cdot)$ is a nonnegative function called radial density, and $c>0$ is a normalization factor such that $f$ integrates to one. The elliptical distributions with location parameter $\mu=\mathbf{0}$ and scale matrix $\Sigma=I_{d \times d}$ are called as spherical distributions [12; $\mathbf{0}$ and $I_{d \times d}$ denote a $d \times 1$ zero vector and a $d \times d$ identity matrix, respectively. Table 1 presents a short selection of prominent spherical distributions, together with their radial densities for univariate case [12.

Table 1: List of selected 1-dimensional spherical distributions.

\begin{tabular}{|c|c|c|c|}
\hline $\begin{array}{l}\text { Probability } \\
\text { distribution }\end{array}$ & Radial density & $\bar{\alpha}_{k, i}$ & $\bar{\alpha}_{i}$ \\
\hline normal & $e^{-\frac{1}{2} u^{2}}$ & $\Psi_{k}^{i}(1)$ & \multirow{6}{*}{$\max _{k=1,2, \ldots, K_{i}} \bar{\alpha}_{k, i}$} \\
\hline$t$ & $\begin{array}{l}\left(1+\frac{1}{\nu} u^{2}\right)^{-(1+\nu) / 2} \\
\nu>0, \nu \text { integer }\end{array}$ & $\Psi_{k}^{i}(1)$ & \\
\hline Cauchy & $\left(1+u^{2}\right)^{-1}$ & $\Psi_{k}^{i}(1)$ & \\
\hline Laplace & $e^{-|u|}$ & $\Psi_{k}^{i}(1)$ & \\
\hline Kotz type & $\begin{array}{l}u^{2(N-1)} e^{-r u^{2 s}} \\
r, s>0, N>\frac{1}{2}\end{array}$ & $\Psi_{k}^{i}\left(\left(\frac{2 N-1}{2 s r}\right)^{\frac{1}{2 s}}\right)$ & \\
\hline $\begin{array}{l}\text { Pearson Type } \\
\text { VII distribution }\end{array}$ & $\begin{array}{l}\left(1+\frac{u^{2}}{m}\right)^{-N} \\
m>0, N>\frac{1}{2}\end{array}$ & $\Psi_{k}^{i}\left(\sqrt{\frac{m}{2 N-1}}\right)$ & \\
\hline
\end{tabular}

\section{Existence of Nash equilibrium under elliptical distribution}

The convexity of the feasibility set of joint chance constraint (2) plays an important role in the existence of Nash equilibrium for the CCG. When there is only one random linear constraint, i.e., $K_{i}=1$, the chance constraint (2) is equivalent to a second-order cone constraint [14]. When the row vectors $A_{k}^{i}, k=1,2, \ldots, K_{i}$, are independent and follow a multivariate normal distribution, then, Strugarek and Henrion [15] showed that the joint chance constraint 22 is convex for large values of $\alpha_{i}$ and Shen et al. 24] proposed a new convex reformulation of (2). Sometimes, in practical situations the distribution of uncertain parameters belongs to the class of elliptical distribution 16. We identify a list of prominent elliptical distributions as given in Table 1 and propose a convex reformulation of $(2)$ when the row vectors $A_{k}^{i}$, 
$k=1,2, \ldots, K_{i}$ are independent and follow $\operatorname{Ellip}_{l_{i}}\left(\mu_{k}^{i}, \Sigma_{k}^{i} ; \varphi_{k}^{i}\right)$ from Table 1 Under independence assumption, the joint chance constraint (2) can be equivalently written as $\prod_{k=1}^{K_{i}} \mathbb{P}\left\{A_{k}^{i} x^{i} \leq b_{k}^{i}\right\} \geq \alpha_{i}$, or alternatively with nonnegative auxiliary variables $z_{k}^{i}[7]$

$$
\left.\begin{array}{c}
\mathbb{P}\left\{A_{k}^{i} x^{i} \leq b_{k}^{i}\right\} \geq \alpha_{i}^{z_{k}^{i}}, k=1,2, \cdots, K_{i}, \\
\sum_{k=1}^{K_{i}} z_{k}^{i}=1, z_{k}^{i} \geq 0, k=1,2, \cdots, K_{i} .
\end{array}\right\}
$$

The chance constraint given in (5) can be treated as an individual chance constraint with $\alpha_{i}^{z_{k}^{i}}$ confidence level. Using the reformulation of individual chance constraint from [14, we have the following reformulation of joint chance constraint (2) 7]

$$
Q_{\alpha_{i}}^{i}=\left\{\begin{array}{l}
(i)\left(\mu_{k}^{i}\right)^{T} x^{i}+\Psi_{k}^{i-1}\left(\alpha_{i}^{z_{k}^{i}}\right)\left\|\left(\Sigma_{k}^{i}\right)^{\frac{1}{2}} x^{i}\right\| \leq b_{k}^{i}, \forall k=1,2, \ldots, K_{i}, \\
(i i) \sum_{k=1}^{K_{i}} z_{k}^{i}=1, \\
(i i i) z_{k}^{i} \geq 0, \forall k=1,2, \ldots, K_{i},
\end{array}\right.
$$

where $\Psi_{k}^{i-1}(\cdot)$ is a quantile function of an 1-dimensional spherical distribution, and $\|\cdot\|$ is the Euclidean norm. The deterministic reformulation (6) of (2) is not convex due to constraints $(i)$ from (6). When $\alpha_{i} \geq 0.5$, we have $\Psi_{k}^{i}-1\left(\alpha_{i}^{z_{k}^{i}}\right) \geq 0$, where $0 \leq z_{k}^{i} \leq 1$. Then, under logarithmic transformation 20] $y_{j}^{i}=\log x_{j}^{i}, j=1,2, \ldots, l_{i}$, we have the following reformulation for (6)

$$
\widetilde{Q}_{\alpha_{i}}^{i}=\left\{\begin{array}{l}
(i)\left(\mu_{k}^{i}\right)^{T} e^{y^{i}}+\|\left(\Sigma_{k}^{i}\right)^{\frac{1}{2}} e^{\log \left(\Psi_{k}^{i}\right)^{-1}\left(\alpha_{i}^{z_{k}^{i}}\right) \mathbb{1}_{l_{i}+y^{i}} \| \leq b_{k}^{i}, \forall k=1,2, \ldots, K_{i},} \\
(i i) \sum_{k=1}^{K_{i}} z_{k}^{i}=1, \\
(i i i) z_{k}^{i} \geq 0, \forall k=1,2, \ldots, K_{i},
\end{array}\right.
$$

where $\mathbb{1}_{l_{i}}$ is an $l_{i} \times 1$ vector of ones, and $e^{y^{i}}=\left(e^{y_{1}^{i}}, \ldots, e^{y_{l_{i}}^{i}}\right)^{T}$ and $e^{\log \left(\Psi_{k}^{i}\right)^{-1}\left(\alpha_{i}^{z_{k}^{i}}\right) \cdot \mathbb{1}_{l_{i}}+y^{i}}=\left(e^{\log \left(\Psi_{k}^{i}\right)^{-1}\left(\alpha_{i}^{z_{k}^{i}}\right)+y_{1}^{i}}, \ldots, e^{\log \left(\Psi_{k}^{i}\right)^{-1}\left(\alpha_{i}^{z_{k}^{i}}\right)+y_{l_{i}}^{i}}\right)^{T}$.

Let $Y^{i}=\left\{y^{i} \in \mathbb{R}^{l_{i}} \mid y_{j}^{i}=\ln x_{j}^{i}, x^{i}=\left(x_{1}^{i}, \ldots, x_{l_{i}}^{i}\right)^{T}, x^{i} \in X^{i}\right\}$. The set $Y^{i}$ is a compact set because it is an image of $X^{i}$ under logarithmic transformation. The convexity might not be preserved under logarithmic transformation. Therefore, we consider only $X^{i}$ for which $Y^{i}$ is a convex set, see for instance 24]. From now onward, we consider the strategy set $X^{i}$ for which $Y^{i}$ is a 
convex set. The reformulation of the feasible strategy set $S_{\alpha_{i}}^{i}$ of player $i, i \in I$, is given by

$$
\widetilde{S}_{\alpha_{i}}^{i}=\left\{\left(y^{i}, z^{i}\right) \in Y^{i} \times \mathbb{R}^{K_{i}} \mid\left(y^{i}, z^{i}\right) \in \widetilde{Q}_{\alpha_{i}}^{i}\right\} .
$$

The convexity of reformulated feasible strategy set $\widetilde{S}_{\alpha_{i}}^{i}$ corresponding to elliptical distributions listed in Table 1 depends on the convexity of function $\log \left(\Psi_{k}^{i}\right)^{-1}\left(\alpha_{i}^{z_{k}^{i}}\right)$ [24]. We show that the function $\log \left(\Psi_{k}^{i}\right)^{-1}\left(\alpha_{i}^{z_{k}^{i}}\right)$ is convex in $z_{k}^{i}$ for probability distributions listed in Table 1 . In order to come-up with the convexity of the set $\widetilde{S}_{\alpha_{i}}^{i}$, we consider the following assumption.

Assumption 2 For each $i \in I$ and $k=1,2, \ldots, K_{i}$, all the components of $\Sigma_{k}^{i}$ and $\mu_{k}^{i}$ are nonnegative.

Lemma 1 Let $\Sigma$ be a $k \times k$ symmetric positive definite matrix with nonnegative entries and $q(\delta)=\left(q_{1}(\delta), q_{2}(\delta), \ldots, q_{k}(\delta)\right)^{T}$ be a $k \times 1$ vector of functions, where each component $q_{j}(\delta)$ is a non-negative convex function of $\delta$. Then, $\left\|\Sigma^{\frac{1}{2}} q(\delta)\right\|$ is a convex function of $\delta$.

Proof The proof follows from the Proposition 2.1 of [14].

Lemma 2 For each $i \in I$ and $k=1,2, \ldots, K_{i}$, let $\left(\Psi_{k}^{i}\right)^{-1}(\cdot)$ be a quantile function of 1-dimensional spherical distribution listed in Table 1. Then, there exists an $\bar{\alpha}_{k, i}$ such that $\log \left(\Psi_{k}^{i}\right)^{-1}\left(\alpha_{i}^{z_{k}^{i}}\right)$ is a convex function of $z_{k}^{i}$ on $[0,1]$ for all $\alpha_{i} \in\left[\bar{\alpha}_{k, i}, 1\right]$.

Proof The proof is given in Appendix A.

Lemma 3 For each $i \in I$, let the convex set $X^{i}$ be such that $Y^{i}$ is a convex set. If Assumption 2 holds for the elliptical distributions listed in Table 1 , $\widetilde{S}_{\alpha_{i}}^{i}$, $i \in I$, defined by (8) is a convex set for all $\alpha_{i} \in\left[\bar{\alpha}_{i}, 1\right] ; \bar{\alpha}_{i}$ is given in Table 1 .

Proof It is evident that $\left(\mu_{k}^{i}\right)^{T} e^{y^{i}}$ is a convex function of $y^{i}$ because the components of $\mu_{k}^{i}$ are nonnegative. From Lemma $2 \log \left(\Psi_{k}^{i}\right)^{-1}\left(\alpha_{i}^{z_{k}^{i}}\right), k=$ $1,2, \ldots, K_{i}$, is a convex function of $z_{k}^{i}$ for all $\alpha_{i} \in\left[\bar{\alpha}_{i}, 1\right]$. Therefore, all the components of the vector of composition functions $e^{\log \left(\Psi_{k}^{i}\right)^{-1}\left(\alpha_{i}^{z_{k}^{i}}\right) \cdot \mathbb{1}_{l_{i}}+y^{i}}$ are nonnegative and convex function of $\left(y^{i}, z_{k}^{i}\right)$. The entries of $\Sigma_{k}^{i}$ are nonnegative. Then, it follows from Lemma $\left[1\right.$ that $\left\|\left(\Sigma_{k}^{i}\right)^{\frac{1}{2}} e^{\log \left(\Psi_{k}^{i}\right)^{-1}\left(\alpha_{i}^{z_{k}^{i}}\right) \mathbb{1}_{l_{i}}+y^{i}}\right\|$ is a convex function of $\left(y^{i}, z_{k}^{i}\right)$. Therefore, the constraints

$$
\left(\mu_{k}^{i}\right)^{T} e^{y^{i}}+\left\|\left(\Sigma_{k}^{i}\right)^{\frac{1}{2}} e^{\log \left(\Psi_{k}^{i}\right)^{-1}\left(\alpha_{i}^{z_{k}^{i}}\right) \mathbb{1}_{l_{i}+y^{i}}}\right\| \leq b_{k}^{i}, \forall k=1,2, \ldots, K_{i},
$$

are convex in $\left(y^{i}, z^{i}\right)$ for all $\alpha_{i} \in\left[\bar{\alpha}_{i}, 1\right]$. The other constraints of $\widetilde{S}_{\alpha_{i}}^{i}$ are convex constraints. Hence, $\widetilde{S}_{\alpha_{i}}^{i}, i \in I$, is a convex set for all $\alpha_{i} \in\left[\bar{\alpha}_{i}, 1\right]$. 
Finally, we show that there exists a Nash equilibrium of a CCG for which we need the following assumption on the players' payoff functions.

Assumption 3 For each player $i, i \in I$, the payoff function $u_{i}\left(\cdot, x^{-i}\right)$ satisfies the non-increasing condition for every $x^{-i} \in X^{-i}$, i.e., for any two points $x^{i}$ and $\bar{x}^{i}$ such that $x_{k}^{i} \leq \bar{x}_{k}^{i}$ for all $k=1,2, \ldots, l_{i}$, we have $u_{i}\left(x^{i}, x^{-i}\right) \geq$ $u_{i}\left(\bar{x}^{i}, x^{-i}\right)$.

Theorem 1 Consider an n-player CCG where

1. Assumptions 1 and 3 holds,

2. the row vectors of $A^{i}, i \in I$ are independent,

3. for each $k=1,2, \ldots K_{i}$, the row vector $A_{k}^{i} \sim \operatorname{Ellip}_{l_{i}}\left(\mu_{k}^{i}, \Sigma_{k}^{i} ; \varphi_{k}^{i}\right)$ w.r.t. Table 1, where the location vector $\mu_{k}^{i}$ and the scale matrix $\Sigma_{k}^{i}$ satisfy Assumption 2.

Then, there exists a Nash equilibrium of a CCG for all $\alpha \in\left[\bar{\alpha}_{1}, 1\right] \times\left[\bar{\alpha}_{2}, 1\right] \times$ $\ldots \times\left[\bar{\alpha}_{n}, 1\right] ; \bar{\alpha}_{i}, i \in I$, is given in Table 1 .

Proof Let $\alpha \in\left[\bar{\alpha}_{1}, 1\right] \times\left[\bar{\alpha}_{2}, 1\right] \times \ldots \times\left[\bar{\alpha}_{n}, 1\right]$. Under Assumption 1, the payoff function $u_{i}\left(x^{i}, x^{-i}\right), i \in I$, is a concave function of $x^{i}$ for every $x^{-i} \in X^{-i}$, and a continuous function of $x$. For each $i \in I$, define a composition function $V_{i}=u_{i} \circ d_{i}$, where $d_{i}: \prod_{i=1}^{n} \mathbb{R}^{l_{i}} \rightarrow \prod_{i=1}^{n} \mathbb{R}_{++}^{l_{i}}$ such that

$$
d_{i}\left(y^{1}, y^{2}, \ldots, y^{n}\right)=\left(e^{y^{1}}, e^{y^{2}}, \ldots, e^{y^{n}}\right) .
$$

Under Assumption 3, $V_{i}\left(y^{i}, y^{-i}\right)$ is a concave function of $y^{i}$ for all $y^{-i}$. The function $V_{i}(y)$ is a continuous function of $y$. It follows from Lemma 3 that the reformulated strategy set $\widetilde{S}_{\alpha_{i}}^{i}$ of player $i, i \in I$, is a convex set. For each $i \in I, \widetilde{S}_{\alpha_{i}}^{i}$ is also a compact set. Then, there exists a Nash equilibrium for an $n$-player non-cooperative game with the payoff functions $V_{i}(y), i \in I$, and the strategy sets $\widetilde{S}_{\alpha_{i}}^{i}, i \in I$ [10, 11]. Therefore, there exists $\left(y^{i *}, z^{i *}\right)_{i \in I}$ such that for each $i \in I$,

$$
V_{i}\left(y^{i *}, y^{-i *}\right) \geq V_{i}\left(y^{i}, y^{-i *}\right), \forall\left(y^{i}, z^{i}\right) \in \widetilde{S}_{\alpha_{i}}^{i} .
$$

For $x^{*}=e^{y^{*}}$ we have for each $i \in I$,

$$
u_{i}\left(x^{i *}, x^{-i *}\right) \geq u_{i}\left(x^{i}, x^{-i *}\right), \forall x^{i} \in S_{\alpha_{i}}^{i} .
$$

Hence, $x^{*}$ is a Nash equilibrium for a CCG for all $\alpha \in\left[\bar{\alpha}_{1}, 1\right] \times\left[\bar{\alpha}_{2}, 1\right] \times \cdots \times$ $\left[\bar{\alpha}_{n}, 1\right]$.

A natural question is whether we can extend Theorem 1 for the entire elliptical family of distributions. The results on the existence of a Nash equilibrium are mainly based on the fixed point arguments of a certain set-valued map, which in our case depends on the convexity of function $\log \left(\Psi_{k}^{i}\right)^{-1}\left(\alpha_{i}^{z_{k}^{i}}\right)$. We give an example of an 1-dimensional spherical distribution for which it is not a convex function. Due to this, the present approach cannot be used to prove the existence of Nash equilibrium for the entire class of elliptical distributions. 
Example 1 Consider an 1-dimensional spherical distribution with probability density function

$$
f(y)= \begin{cases}\frac{3}{2} y^{2}, & y \in[-1,1] \\ 0, & \text { otherwise }\end{cases}
$$

The cumulative distribution function $\Psi$ can be expressed as

$$
\Psi(y)= \begin{cases}0, & (-\infty,-1) \\ \frac{1}{2} y^{3}+\frac{1}{2}, & y \in[-1,1] \\ 1, & (1,+\infty)\end{cases}
$$

Let $h(z)=\log (\Psi)^{-1}\left(\alpha^{z}\right)$. We have $h(z)=\frac{1}{3} \log \left(2 \alpha^{z}-1\right)$. For $\alpha>\frac{1}{2}$, the second derivative of $h(z)$ is given by

$$
h^{\prime \prime}(z)=\frac{2}{3}(\log \alpha)^{2} \alpha^{z}\left(\frac{-1}{\left(2 \alpha^{z}-1\right)^{2}}\right) .
$$

It is easy to see that $h^{\prime \prime}(z)<0$. This implies that $\log (\Psi)^{-1}\left(\alpha^{z}\right)$ is not a convex function.

\section{Existence of Nash equilibrium under a finite mixture of elliptical distributions}

We assume that the probability distribution of row vector $A_{k}^{i}, k=1,2, \ldots, K_{i}$, is a finite mixture of elliptical distributions listed in Table 11. The density function $f_{k}^{i}(\mathbf{u})$ of $A_{k}^{i}$ is defined by

$$
f_{k}^{i}(\mathbf{u})=\sum_{m=1}^{M_{k}^{i}} w_{k, m}^{i} f_{k, m}^{i}(\mathbf{u})
$$

where $f_{k, m}^{i}(\mathbf{u})$ is the density function of an elliptical distribution from Table 1 with location parameter $\mu_{k, m}^{i}$, scale matrix $\Sigma_{k, m}^{i}$, and characteristic generator $\varphi_{k, m}^{i}$. In addition, $\left(w_{k, m}^{i}\right)_{m=1}^{M_{k}^{i}}$ is a weight vector such that $w_{k, m}^{i} \geq 0$ for all $m=1,2, \ldots, M_{k}^{i}$, and $\sum_{m=1}^{M_{k}^{i}} w_{k, m}^{i}=1$. From the definition of the mixture distribution, we have

$$
\mathbb{P}\left\{A_{k}^{i} x^{i} \leq b_{k}^{i}\right\}=\int_{A_{k}^{i} x^{i} \leq b_{k}^{i}} f_{k}^{i}\left(A_{k}^{i}\right) d A_{k}^{i}=\sum_{m=1}^{M_{k}^{i}} w_{k, m}^{i} \int_{A_{k}^{i} x^{i} \leq b_{k}^{i}} f_{k, m}^{i}\left(A_{k}^{i}\right) d A_{k}^{i},
$$

In this case, the joint chance constraint (2) can be reformulated as

$$
\left\{\begin{array}{l}
\sum_{m=1}^{M_{k}^{i}} w_{k, m}^{i} \mathbb{P}_{\sim P_{m}}\left\{A_{k}^{i} x^{i} \leq b_{k}^{i}\right\} \geq \alpha_{i}^{z_{k}^{i}}, k=1,2, \ldots, K_{i} \\
\sum_{k=1}^{K_{i}} z_{k}^{i}=1, \quad z_{k}^{i} \geq 0, k=1,2, \ldots, K_{i}
\end{array}\right.
$$


where $\mathbb{P}_{\sim P_{m}}\{\cdot\}$ denotes the probability function of the $m$ th probability distribution $P_{m}$. We introduce an auxiliary variables $\eta_{k, m}^{i}, m=1,2, \ldots, M_{k}^{i}$, $k=1,2, \ldots, K_{i}$, and reformulate the constraint (11) as follows

$$
\left\{\begin{array}{l}
\mathbb{P}_{\sim P_{m}}\left\{A_{k}^{i} x^{i} \leq b_{k}^{i}\right\} \geq \eta_{k, m}^{i}, m=1,2, \ldots, M_{k}^{i}, k=1,2, \ldots, K_{i}, \\
\sum_{m=1}^{M_{k}^{i}} w_{k, m}^{i} \eta_{k, m}^{i} \geq \alpha_{i}^{z_{k}^{i}}, k=1,2, \ldots, K_{i} \\
\sum_{k=1}^{K_{i}} z_{k}^{i}=1, \quad z_{k}^{i} \geq 0, k=1,2, \ldots, K_{i},
\end{array}\right.
$$

which is equivalent to

$$
\left\{\begin{array}{l}
\left(\mu_{k, m}^{i}\right)^{T} x^{i}+\left(\Psi_{k, m}^{i}\right)^{-1}\left(\eta_{k, m}^{i}\right)\left\|\left(\Sigma_{k, m}^{i}\right)^{\frac{1}{2}} x^{i}\right\| \leq b_{k}^{i}, \\
m=1,2, \ldots, M_{k}^{i}, k=1,2, \ldots, K_{i}, \\
\sum_{m=1}^{M_{k}^{i}} w_{k, m}^{i} \eta_{k, m}^{i} \geq \alpha_{i}^{z_{k}^{i}}, k=1,2, \ldots, K_{i}, \\
\sum_{k=1}^{K_{i}} z_{k}^{i}=1, \quad z_{k}^{i} \geq 0, k=1,2, \ldots, K_{i},
\end{array}\right.
$$

where $\left(\Psi_{k, m}^{i}\right)^{-1}(\cdot)$ is a quantile function of one dimensional spherical distribution. By taking $r_{k, m}^{i}=\left(\Psi_{k, m}^{i}\right)^{-1}\left(\eta_{k, m}^{i}\right)$, we have the following reformulation of joint chance constraint (2)

$$
Q_{\alpha_{i}}^{i}=\left\{\begin{array}{l}
(i)\left(\mu_{k, m}^{i}\right)^{T} x^{i}+r_{k, m}^{i}\left\|\left(\sum_{k, m}^{i}\right)^{\frac{1}{2}} x^{i}\right\| \leq b_{k}^{i}, \\
\quad m=1,2, \ldots, M_{k}^{i}, k=1,2, \ldots, K_{i}, \\
(i i) \sum_{m=1}^{M_{k}^{i}} w_{k, m}^{i} \Psi_{k, m}^{i}\left(r_{k, m}^{i}\right) \geq \alpha_{i}^{z_{k}^{i}}, k=1,2, \ldots, K_{i}, \\
(i i i) \sum_{k=1}^{K_{i}} z_{k}^{i}=1, \\
(i v) z_{k}^{i} \geq 0, k=1,2, \ldots, K_{i} .
\end{array}\right.
$$

The reformulation 14 is not convex in the new variables $\left(x^{i},\left(r_{k, m}^{i}\right)_{k=1, m=1}^{K_{i}, M_{k}^{i}},\left(z_{k}^{i}\right)_{k=1}^{K_{i}}\right)$. To apply the logarithmic transformation as in the previous case, it is necessary to have $r_{k, m}^{i}>0$ for all $k$ and $m$. Next, we show that for each $k$ and $m, r_{k, m}^{i}>0$, under certain conditions on weight vectors and confidence level.

Lemma 4 For a given $\alpha_{i} \in[0,1]$ and for each $m=1,2, \ldots, M_{k}^{i}$ and $k=$ $1,2, \ldots, K_{i}$ if $w_{k, m}^{i} \geq \frac{1}{1-C}\left(1-\alpha_{i}\right)$ such that $\sum_{m=1}^{M_{k}^{i}} w_{k, m}^{i}=1$, where $\frac{1}{2}<$ $C<1$, then $r_{k, m}^{i} \geq\left(\Psi_{k, m}^{i}\right)^{-1}(C)>0$. 
Proof For each $k$ and $m$, it follows from constraint (ii) of 14 that

$$
1-w_{k, m}^{i}+w_{k, m}^{i} \Psi_{k, m}^{i}\left(r_{k, m}^{i}\right) \geq \alpha_{i}^{z_{k}^{i}}
$$

Therefore,

$$
\Psi_{k, m}^{i}\left(r_{k, m}^{i}\right) \geq 1-\frac{1-\alpha_{i}^{z_{k}^{i}}}{w_{k, m}^{i}}
$$

For $0 \leq z_{k}^{i} \leq 1$, we have $\alpha_{i}^{z_{k}^{i}} \geq \alpha_{i}$. Then, under the condition on the weight vector, we have $r_{k, m}^{i} \geq\left(\Psi_{k, m}^{i}\right)^{-1}(C)>0$.

For the elliptical distributions listed in Table 1, we derive the bounds on confidence level and weights for which the condition of Lemma 4 is satisfied. Let $w_{k, m}^{i} \in\left[\bar{w}_{i}, 1\right]$ for all $k$ and $m$, where $\bar{w}_{i}$ is defined as in the third column of Table 2 Such a choice of $w_{k, m}^{i}$ does not need to form a weight vector for an arbitrary choice of $\alpha_{i}$. The condition $0 \leq w_{k, m}^{i} \leq 1$ gives $\alpha_{i} \geq 1-\frac{1}{\beta_{k, m}^{i}}$, and the condition $\sum_{m=1}^{M_{k}^{i}} w_{k, m}^{i}=1$ leads to $\alpha_{i} \geq 1-\frac{1}{\sum_{m=1}^{M_{k}^{i}} \beta_{k, m}^{i}}$. The uniform lower bound $\bar{\alpha}_{i}$ on $\alpha_{i}$ can be obtained by taking into account all the constraints and it is given in the last column of Table 2 . Therefore, for $\alpha_{i} \in\left[\bar{\alpha}_{i}, 1\right]$ and $w_{k, m}^{i} \in\left[\bar{w}_{i}, 1\right]$ for all $k$ and $m$, it is easy to see that the condition of Lemma 4 is satisfied. Therefore, $r_{k, m}^{i}>0$ for all $k$ and $m$.

Table 2: Bounds for confidence level and weights used in the mixture

\begin{tabular}{|c|c|c|c|}
\hline $\begin{array}{l}\text { Probability } \\
\text { distribution }\end{array}$ & $\beta_{k, m}^{i}$ & $\bar{w}_{i}$ & $\bar{\alpha}_{i}$ \\
\hline normal & $\frac{1}{1-\Psi_{k, m}^{i}(1)}$ & \multirow{6}{*}{$\max _{\substack{k=1,2, \ldots, K_{i}, m=1,2, \ldots, M_{k}^{i}}}\left\{\beta_{k, m}^{i}\left(1-\alpha_{i}\right)\right\}$} & \multirow{6}{*}{$1-\frac{1}{\sum_{m=1}^{M_{k}^{i}} \beta_{k, m}^{i}}$} \\
\hline$t$ & $\frac{1}{1-\Psi_{k, m}^{i}(1)}$ & & \\
\hline Cauchy & $\frac{1}{1-\Psi_{k, m}^{i}(1)}$ & & \\
\hline Laplace & $\frac{1}{1-\Psi_{k, m}^{i}(1)}$ & & \\
\hline Kotz type & $\frac{1}{1-\Psi_{k, m}^{i}\left(\left(\frac{2 N-1}{2 s r}\right)^{\frac{1}{2 s}}\right)}$ & & \\
\hline $\begin{array}{l}\text { Pearson Type } \\
\text { VII }\end{array}$ & $\frac{1}{1-\Psi_{k, m}^{i}\left(\sqrt{\frac{m}{2 N-1}}\right)}$ & & \\
\hline
\end{tabular}
distribution

Now, we reformulate (14) by using the change of variables under logarithmic transformation. We transform the vector $x^{i} \in X^{i}$ into a vector $y^{i} \in \mathbb{R}^{l_{i}}$, where $y_{j}^{i}=\ln x_{j}^{i}, j=1,2, \ldots, l_{i}$, and $\tau_{k, m}^{i}=\ln r_{k, m}^{i}, m=1,2, \ldots, M_{k}^{i}, k=$ 
$1,2, \ldots, K_{i}$. We have the following reformulation of 14

$$
\widetilde{Q}_{\alpha_{i}}^{i}=\left\{\begin{array}{l}
(i)\left(\mu_{k, m}^{i}\right)^{T} e^{y^{i}}+\|\left(\sum_{k, m}^{i}\right)^{\frac{1}{2}} e^{y^{i}+\tau_{k, m}^{i} \cdot \mathbb{1}_{l_{i}} \| \leq b_{k}^{i},} \\
m=1,2, \ldots, M_{k}^{i}, k=1,2, \ldots, K_{i}, \\
(i i) \sum_{m=1}^{M_{k}^{i}} w_{k, m}^{i} \Psi_{k, m}^{i}\left(e^{\tau_{k, m}^{i}}\right) \geq \alpha_{i}^{z_{k}^{i}}, k=1,2, \ldots, K_{i}, \\
(i i i) \sum_{k=1}^{K_{i}} z_{k}^{i}=1, \\
(i v) z_{k}^{i} \geq 0, k=1,2, \ldots, K_{i},
\end{array}\right.
$$

The reformulation of the feasible strategy set $S^{i}\left(\alpha_{i}\right)$ of player $i, i \in I$, is given by

$$
\begin{aligned}
\widetilde{S}_{\alpha_{i}}^{i}=\left\{\left(y^{i}, z^{i}, \tau_{1}^{i}, \ldots, \tau_{K_{i}}^{i}\right) \in Y^{i} \times \mathbb{R}_{+}^{K_{i}} \times \mathbb{R}^{M_{1}^{i}} \times \cdots \times \mathbb{R}^{M_{K_{i}}^{i}} \mid\right. \\
\left.\left(y^{i}, z^{i}, \tau_{1}^{i}, \ldots, \tau_{K_{i}}^{i}\right) \in \widetilde{Q}_{\alpha_{i}}^{i}\right\} .
\end{aligned}
$$

It is easy to see that $\widetilde{S}_{\alpha_{i}}^{i}$ is a compact set. As similar to Assumption 2 , we have the following assumption on the location and scale parameters of the elliptical distributions used in the mixture.

Assumption 4 For each $i \in I, m=1,2, \ldots, M_{k}^{i}, k=1,2, \ldots, K_{i}$, all the components of $\Sigma_{k, m}^{i}$ and $\mu_{k, m}^{i}$ are nonnegative.

To prove $\widetilde{S}_{\alpha_{i}}^{i}$ a convex set for a given $\alpha_{i}$, it is enough to show that the constraints $(i)$ and $(i i)$ of $(15)$ are convex.

Lemma 5 For each $i \in I$, suppose $\alpha_{i} \in\left[\bar{\alpha}_{i}, 1\right]$ and $w_{k, m}^{i} \in\left[\bar{w}_{i}, 1\right]$ for all $k=1,2, \ldots, K_{i}$ and $m=1,2, \ldots, M_{k}^{i}$, where $\bar{\alpha}_{i}$ and $\bar{w}_{i}$ are given as in Table 2. If $\Psi_{k, m}^{i}(\cdot)$ is one dimensional distribution function of spherical distributions listed in Table 2, $\Psi_{k, m}^{i}\left(e^{\tau_{k, m}^{i}}\right)$ is a concave function of $\tau_{k, m}^{i}$.

Proof The proof is given in Appendix B

Lemma 6 For each $i \in I$, let the convex set $X^{i}$ be such that $Y^{i}$ is a convex set. Suppose that the probability distributions used in the mixture are from the list given in Table 2. Then, under Assumption 4, the strategy set $\widetilde{S}_{\alpha_{i}}^{i}$, $i \in I$, defined by 116 is a convex set for all $\alpha_{i} \in\left[\bar{\alpha}_{i}, 1\right]$ and $w_{k, m}^{i} \in\left[\bar{w}_{i}, 1\right]$, $m=1,2, \ldots, M_{k}^{i}, k=1,2, \ldots, K_{i} ; \bar{\alpha}_{i}$ and $\bar{w}_{i}$ are given in Table 2 .

Proof As a nonnegative linear combination of concave function is a concave function, it follows from Lemma 5 that $\sum_{m=1}^{M_{k}^{i}} w_{k, m}^{i} \Psi_{k, m}^{i}\left(e^{\tau_{k, m}^{i}}\right)-\alpha_{i}^{z_{k}^{i}}$ is a concave function of $\left(\left(\tau_{k, m}^{i}\right)_{m=1}^{M_{k}^{i}}, z_{k}^{i}\right)$. Hence, for each $k=1,2, \ldots, K_{i}$, the constraint (ii) of 15 is a convex constraint. Under Assumption 4 , it follows 


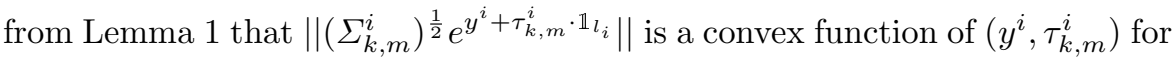
each $k$ and each $m$. For each $k$ and each $m,\left(\mu_{k, m}^{i}\right)^{T} e^{y^{i}}$ is a convex function of $y^{i}$ because $\mu_{k, m}^{i} \geq 0$. Therefore, for each $k$ and each $m$ the constraint $(i)$ of 15 is a convex constraint. It is easy to see that the other constraints of $\widetilde{S}_{\alpha_{i}}^{i}$ are convex constraints. Therefore, $\widetilde{S}_{\alpha_{i}}^{i}$ is a convex set.

Finally, thanks to the convex reformulation of feasible strategy set for each player, we show that there exists a Nash equilibrium.

Theorem 2 Consider an n-player CCG where

1. Assumptions 1 and 3 holds,

2. the row vectors of $A^{i}, i \in I$ are independent,

3. for each $k=1,2, \ldots K_{i}$, the row vector $A_{k}^{i}, i \in I$, follows a finite mixture of multivariate elliptical distributions listed in the Table 2, where the location vector $\mu_{k, m}^{i}$ and the scale matrix $\Sigma_{k, m}^{i}$ of the mth elliptical distribution satisfy Assumption 4 .

4. for each $i \in I$, the weights $w_{k, m}^{i} \in\left[\bar{w}_{i}, 1\right], k=1,2, \ldots K_{i}, m=1,2, \ldots, M_{k}^{i}$, where $\bar{w}_{i}$ is given in Table 2

Then, there exists a Nash equilibrium of the CCG for all $\alpha \in\left[\bar{\alpha}_{1}, 1\right] \times\left[\bar{\alpha}_{2}, 1\right] \times$ $\cdots \times\left[\bar{\alpha}_{n}, 1\right] ; \bar{\alpha}_{i}, i \in I$, is given in Table 2 .

Proof It follows from Lemma 6 that the reformulated strategy set $\widetilde{S}_{\alpha_{i}}^{i}, i \in I$, is convex for all $\alpha \in\left[\bar{\alpha}_{i}, 1\right]$. Then, the proof follows from the similar arguments used in Theorem 1 .

\section{Competition in financial market}

We consider the case of two investment firms which compete on the same market. Each firm invests in the same set of portfolios consisting of different assets. Let $J$ be the set of portfolios and $\mathcal{A}_{k}$ be the set of assets in the portfolio $k$. We assume that the portfolios are disjoint, i.e., for any $k, l \in J, \mathcal{A}_{k}$ and $\mathcal{A}_{l}$ are disjoint sets. Each firm decides to invest its money in different portfolios whose returns are defined by random vectors. Let $x_{k}^{i}=\left(x_{k j}^{i}\right)_{j \in \mathcal{A}_{k}}$ be the investment vector of firm $i, i=1,2$, for $k$ th portfolio; $x_{k j}^{i}$ represents the amount of money firm $i$, invested in the $j$ th asset of $k$ th portfolio. Let $x^{i}=$ $\left(x_{k}^{i}\right)_{k \in J}$ denotes an investment vector of firm $i$. The set of investments $X^{i}$ of firm $i$ is defined as

$$
X^{i}=\left\{x^{i} \mid \sum_{j \in \mathcal{A}_{k}} x_{k j}^{i} \leq W_{k}^{i}, x_{k j}^{i} \geq \varepsilon_{i}, \forall k \in J, j \in \mathcal{A}_{k}\right\},
$$

where $\varepsilon_{i}>0$ is the minimum amount invested in any asset by firm $i$ and $W_{k}^{i}$ denotes the maximum amount that can be invested by firm $i$ in portfolio $k$. The vector $x=\left(x^{1}, x^{2}\right) \in X^{1} \times X^{2}$ represents an investment profile of 
both the firms. Let $R_{k}^{i}=\left(R_{k j}^{i}\right)_{j \in \mathcal{A}_{k}}$ be a random return vector of firm $i$ from portfolio $k$. For a given investment vector $x_{k}^{i}$, the total return received by firm $i$ from portfolio $k$ is defined as $\left(x_{k}^{i}\right)^{T} R_{k}^{i}$ and $\left(-x_{k}^{i}\right)^{T} R_{k}^{i}$ represents random loss incurred by firm $i$ (negative return) from portfolio $k$. Let $d_{k}^{i}$ be the maximal allowable loss level of firm $i$ from portfolio $k$. Firm $i$ wants to keep its random losses below the maximal allowable level with at least $\alpha_{i}$ probability, i.e.,

$$
P\left\{\left(-x_{k}^{i}\right)^{T} R_{k}^{i} \leq d_{k}^{i}, k \in J\right\} \geq \alpha_{i}, \forall i=1,2 .
$$

Therefore, the set of feasible investments of firm $i$ is given by

$$
S_{\alpha_{i}}^{i}=\left\{x^{i} \in X^{i} \mid P\left\{\left(-x_{k}^{i}\right)^{T} R_{k}^{i} \leq d_{k}^{i}, k \in J\right\} \geq \alpha_{i}\right\} .
$$

We consider the case where each firm incurs transaction costs. The transaction costs can be used to model a number of costs involved in a financial market, e.g., brokerage fees, bid-ask spread and market impact [19, 21]. Usually the transaction costs are higher for popular assets due to the participation of many firms and it is directly proportional to the money invested in it. Therefore, the transaction cost incurred by a firm also depends on the investment of other firms. In this paper, we consider the quadratic transaction costs, i.e., for a given investment profile $\left(x_{k}^{1}, x_{k}^{2}\right)$ for portfolio $k$, the firm 1 incurs cost $c_{k}^{1}\left(x_{k}^{1}\right)^{T}\left(x_{k}^{1}+x_{k}^{2}\right)$ and firm 2 incurs cost $c_{k}^{2}\left(x_{k}^{2}\right)^{T}\left(x_{k}^{1}+x_{k}^{2}\right)$ to manage portfolio $k$. Then, for a given investment profile $x=\left(x^{1}, x^{2}\right)$, the payoff function of firm $i, i=1,2$, is given by

$$
u_{i}\left(x^{1}, x^{2}\right)=\sum_{k \in J}\left(\mathbb{E}\left[R_{k}^{i}\right]\right)^{T} x_{k}^{i}-\sum_{k \in J} c_{k}^{i}\left(x_{k}^{i}\right)^{T}\left(x_{k}^{1}+x_{k}^{2}\right) .
$$

Under the logarithmic transformation, the payoff functions of firm 1 and firm 2 , are given by

$$
\begin{aligned}
& V_{1}\left(y^{1}, y^{2}\right)=\sum_{k \in J}\left(\mathbb{E}\left[R_{k}^{1}\right]-c_{k}^{1} e^{y_{k}^{2}}\right)^{T} e^{y_{k}^{1}}-\sum_{k \in J} c_{k}^{1} \sum_{j \in \mathcal{A}_{k}} e^{2 y_{k j}^{1},} \\
& V_{2}\left(y^{1}, y^{2}\right)=\sum_{k \in J}\left(\mathbb{E}\left[R_{k}^{2}\right]-c_{k}^{2} e^{y_{k}^{1}}\right)^{T} e^{y_{k}^{2}}-\sum_{k \in J} c_{k}^{2} \sum_{j \in \mathcal{A}_{k}} e^{2 y_{k j}^{2},}
\end{aligned}
$$

where $e^{y_{k}^{i}}=\left(e^{y_{k j}^{i}}\right)_{j \in \mathcal{A}_{k}}, i=1,2$.

For a fixed $y^{2}$, the function $V_{1}\left(y^{1}, y^{2}\right)$ is a concave function of $y^{1}$ if its Hessian matrix is negative semidefinite. Therefore, $V_{1}\left(y^{1}, y^{2}\right)$ is a concave function of $y^{1}$ for a given $y^{2}$ if

$$
4 c_{k}^{1} e^{y_{k j}^{1}}+c_{k}^{1} e^{y_{k j}^{2}} \geq \mathbb{E}\left[R_{k j}^{1}\right], j \in \mathcal{A}_{k}, k \in J .
$$

Similarly, $V_{2}\left(y^{1}, y^{2}\right)$ is a concave function of $y^{2}$ for a given $y^{1}$ if

$$
4 c_{k}^{2} e^{y_{k j}^{2}}+c_{k}^{2} e^{y_{k j}^{1}} \geq \mathbb{E}\left[R_{k j}^{2}\right], j \in \mathcal{A}_{k}, k \in J .
$$

The conditions (18) and (19) are satisfied for suitable choice of the minimum invested amount $\varepsilon_{i}$ of firm $i$. The mixture of elliptical distributions are often 
used in the literature to model the random return vectors in financial market [3, 13, 17. We assume that the random return vectors $R_{k}^{i}, k \in J$, are independent and follow a mixture of elliptical distributions. Then, under logarithmic transformation the convex reformulation $\tilde{S}_{\alpha_{i}}^{i}$ of $(17)$ is given by $(16)$, where

$$
Y^{i}=\left\{y^{i} \mid \sum_{j \in \mathcal{A}_{k}} e^{y_{k j}^{i}} \leq W_{k}^{i}, y_{k j}^{i} \geq \ln \left(\varepsilon_{i}\right), \forall k \in J, j \in \mathcal{A}_{k}\right\} \text {. }
$$

If the conditions 18$)$ and $(19)$ are satisfied, it follows from Theorem 2 that there exists a Nash equilibrium of the game.

\subsection{Best response algorithm}

In this section, we present a best response algorithm which is used to compute the Nash equilibria of the above game problem. For a fixed $\left(y^{2}, z^{2},\left(\tau_{k}^{2}\right)_{k \in J}\right) \in$ $\tilde{S}_{\alpha_{2}}^{2}$, player 1 solves the following convex optimization problem

$$
\begin{aligned}
& {\left[P_{1}\right] \max _{y^{1}, z^{1},\left(\tau_{k}^{1}\right)_{k \in J}} V_{1}\left(y^{1}, y^{2}\right)} \\
& \text { s.t. }\left(y^{1}, z^{1},\left(\tau_{k}^{1}\right)_{k \in J}\right) \in \widetilde{S}_{\alpha_{1}}^{1} .
\end{aligned}
$$

The set of optimal solution of $\left[P_{1}\right]$, which is also called the best response set of player 1 , is given by

$$
B R_{1}\left(y^{2}\right)=\left\{\left(\bar{y}^{1}, \bar{z}^{1},\left(\bar{\tau}_{k}^{1}\right)_{k \in J}\right) \mid V_{1}\left(\bar{y}^{1}, y^{2}\right) \geq V_{1}\left(y^{1}, y^{2}\right), \forall\left(y^{1}, z^{1},\left(\tau_{k}^{1}\right)_{k \in J}\right) \in \widetilde{S}_{\alpha_{1}}^{1}\right\} .
$$

Similarly, for a fixed $\left(y^{1}, z^{1},\left(\tau_{k}^{1}\right)_{k \in J}\right) \in \tilde{S}_{\alpha_{1}}^{1}$, player 2 solves the following convex optimization problem

$$
\begin{aligned}
& {\left[P_{2}\right] \underset{y^{2}, z^{2},\left(\tau_{k}^{2}\right)_{k \in J}}{\max } V_{2}\left(y^{1}, y^{2}\right)} \\
& \text { s.t. }\left(y^{2}, z^{2},\left(\tau_{k}^{2}\right)_{k \in J}\right) \in \widetilde{S}_{\alpha_{2}}^{2} .
\end{aligned}
$$

The best response set of player 2 , is given by

$$
B R_{2}\left(y^{1}\right)=\left\{\left(\bar{y}^{2}, \bar{z}^{2},\left(\bar{\tau}_{k}^{2}\right)_{k \in J}\right) \mid V_{2}\left(y^{1}, \bar{y}^{2}\right) \geq V_{2}\left(y^{1}, y^{2}\right), \forall\left(y^{2}, z^{2},\left(\tau_{k}^{2}\right)_{k \in J}\right) \in \widetilde{S}_{\alpha_{2}}^{2}\right\} .
$$

It is clear that, if $\left(y^{1 *}, z^{1 *},\left(\tau_{k}^{1 *}\right)_{k \in J}\right) \in B R_{1}\left(y^{2 *}\right)$ and $\left(y^{2 *}, z^{2 *},\left(\tau_{k}^{2 *}\right)_{k \in J}\right) \in$ $B R_{2}\left(y^{1 *}\right),\left(x^{1 *}, x^{2 *}\right)=\left(e^{y^{1 *}}, e^{y^{2 *}}\right)$ is a Nash equilibrium of the game. For computational purpose, we use the best response algorithm as outlined below:

Algorithm 1 (Best response algorithm) Step-1 Select initial feasible point $\left.\left(y^{2(0)}, z^{2(0)},\left(\tau_{k}^{2(0)}\right)_{k \in J}\right)\right) \in \widetilde{S}_{\alpha_{2}}^{2}$ for player 2. Set $n:=0$.

Step-2 Solve convex optimization problem $\left[P_{1}\right]$ and find a point $\left(y^{1(n)}, z^{1(n)},\left(\tau_{k}^{1(n)}\right)_{k \in J}\right) \in B R_{1}\left(y^{2(n)}\right)$. 
Step-3 If $\left(y^{2(n)}, z^{2(n)},\left(\tau_{k}^{2(n)}\right)_{k \in J}\right) \in B R_{1}\left(y^{1(n)}\right)$, then set $\left(x^{1 *}, x^{2 *}\right)=$ $\left(e^{y^{1(n)}}, e^{y^{2(n)}}\right)$ and stop. Otherwise, solve convex optimization problem $\left[P_{2}\right]$ and find a point $\left(y^{2(n)}, z^{2(n)},\left(\tau_{k}^{2(n)}\right)_{k \in J}\right) \in B R_{1}\left(y^{1(n)}\right)$, set $n=n+1$ and go to step 2.

If the Algorithm 1 stops, $\left(x^{1 *}, x^{2 *}\right)$ is a Nash equilibrium of the game. The proof that Algorithm 1 never cycles is still an open problem.

\subsection{Case Study}

Example 2 We consider two investment firms with two portfolios where each portfolio consists of three assets, i.e., $J=\{1,2\}$ and $\mathcal{A}_{k}=\{1,2,3\}, k \in J$. We take the confidence level values of firm 1 and firm 2 as 0.95, i.e., $\alpha_{1}=$ $\alpha_{2}=0.95$. The probability distribution of the return vector $R_{k}^{i}$ is assumed to be an equiprobable mixture of two normal distributions $\mathcal{N}\left(\mu_{k, 1}^{i}, \Sigma_{k, 1}^{i}\right)$ and $\mathcal{N}\left(\mu_{k, 2}^{i}, \Sigma_{k, 2}^{i}\right)$, where $\mu_{k, m}^{i}$ is generated uniformly on $[0.1,0.3]^{3}$, and $\Sigma_{k, m}^{i}$ is generated randomly with each element drawn from $[0,0.1]$ uniformly. The randomly generated mean vectors and covariance matrices are summarized as follows:

$$
\begin{aligned}
& \mu_{1,1}^{1}=\left(\begin{array}{l}
0.1131 \\
0.1995 \\
0.2909
\end{array}\right), \mu_{1,2}^{1}=\left(\begin{array}{c}
0.2991 \\
0.1587 \\
0.1081
\end{array}\right), \mu_{2,1}^{1}=\left(\begin{array}{c}
0.1092 \\
0.2006 \\
0.2921
\end{array}\right), \mu_{2,2}^{1}=\left(\begin{array}{c}
0.2967 \\
0.2022 \\
0.1045
\end{array}\right) \text {. } \\
& \mu_{1,1}^{2}=\left(\begin{array}{c}
0.1103 \\
0.2022 \\
0.2931
\end{array}\right), \mu_{1,2}^{2}=\left(\begin{array}{c}
0.2896 \\
0.2011 \\
0.1052
\end{array}\right), \mu_{2,1}^{2}=\left(\begin{array}{l}
0.1048 \\
0.1988 \\
0.2957
\end{array}\right), \mu_{2,2}^{2}=\left(\begin{array}{l}
0.2997 \\
0.2053 \\
0.1052
\end{array}\right) \text {. } \\
& \Sigma_{1,1}^{1}=\left(\begin{array}{ccc}
0.1 & 0.0927 & 0.0202 \\
0.0927 & 0.1 & 0.0187 \\
0.0202 & 0.0187 & 0.1
\end{array}\right), \Sigma_{1,2}^{1}=\left(\begin{array}{ccc}
0.1 & 0.0379 & 0.0520 \\
0.0379 & 0.1 & 0.0473 \\
0.052 & 0.0473 & 0.1
\end{array}\right) \\
& \Sigma_{2,1}^{1}=\left(\begin{array}{ccc}
0.1 & 0.0589 & 0.0382 \\
0.0589 & 0.1 & 0.0554 \\
0.0382 & 0.0554 & 0.1
\end{array}\right), \Sigma_{2,2}^{1}=\left(\begin{array}{ccc}
0.1 & 0.0072 & 0.0250 \\
0.0072 & 0.1 & 0.0476 \\
0.025 & 0.0476 & 0.1
\end{array}\right) \text {. } \\
& \Sigma_{1,1}^{2}=\left(\begin{array}{ccc}
0.1 & 0.0612 & 0.0049 \\
0.0612 & 0.1 & 0.0732 \\
0.0049 & 0.0732 & 0.1
\end{array}\right), \Sigma_{1,2}^{2}=\left(\begin{array}{ccc}
0.1 & 0.031 & 0.0421 \\
0.031 & 0.1 & 0.0404 \\
0.0421 & 0.0404 & 0.1
\end{array}\right) \\
& \Sigma_{2,1}^{2}=\left(\begin{array}{ccc}
0.1 & 0.0469 & 0.0245 \\
0.0469 & 0.1 & 0.0167 \\
0.0245 & 0.0167 & 0.1
\end{array}\right), \Sigma_{2,2}^{2}=\left(\begin{array}{ccc}
0.1 & 0.023 & 0.0522 \\
0.023 & 0.1 & 0.0384 \\
0.0522 & 0.0384 & 0.1
\end{array}\right) \text {. }
\end{aligned}
$$

The values of other parameters are given by $\varepsilon_{1}=0.3, \varepsilon_{2}=0.25, d_{k}^{1}=$ $0.75, d_{k}^{2}=0.6, W_{k}^{1}=W_{k}^{2}=1, k \in J$ and $c_{k}^{1}=c_{k}^{2}=0.2, k \in J$. 
We compute the Nash equilibrium of the game using the Algorithm 1. We run the numerical experiments on an Intel Core i7-8550U CPU $1.80 \mathrm{GHz} 2.00 \mathrm{GHz}$ with 16.0 GB RAM machine. To solve the best response convex optimization problem of both firms, we use CVX package in MATLAB. For the above data the algorithm converges to a Nash equilibrium point $\left(x^{1 *}, x^{2 *}\right)$ given by

$$
\begin{gathered}
x^{1 *}=((0.3478,0.3,0.3249),(0.3431,0.3319,0.3250)), \\
x^{2 *}=((0.3385,0.3067,0.3390),(0.3333,0.3314,0.3353)) .
\end{gathered}
$$

Figure 1 shows that the best response algorithm converges to a Nash equi-

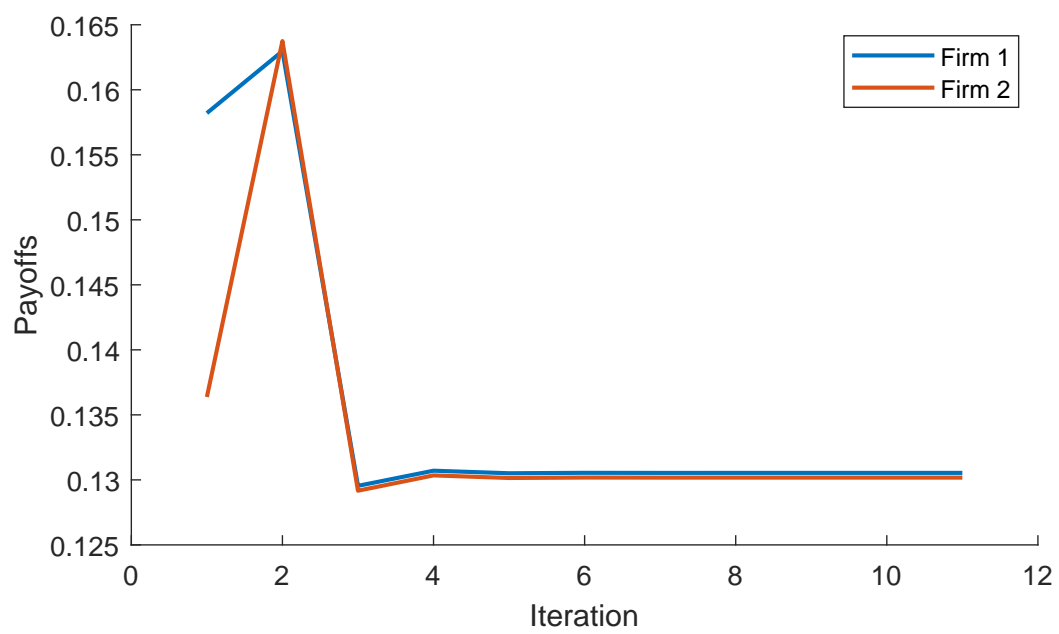

Fig. 1: Convergence of Nash equilibrium payoff

librium payoffs of both the firms after few iterations. The total CPU time to compute Nash equilibrium is 23.12 seconds.

We also perform numerical experiments for a relatively large size model with 10 sets of portfolios and 20 assets in each portfolio. The distribution of $R_{k}^{i}$ is considered as an equiprobable mixture of two normal distributions whose mean vectors and covariance matrices are generated similar to the Example 2. The parameters $\varepsilon_{1}$ and $\varepsilon_{2}$ are generated uniformly on the interval [0.2,0.3], and $W_{k}^{1}=W_{k}^{2}=10, c_{k}^{1}=c_{k}^{2}=0.2$ for all $k \in J$, and $d_{k}^{1}, d_{k}^{2}, k \in J$, are drawn randomly from the interval $[5,8]$, and $\alpha_{1}=\alpha_{2}=0.95$. We consider 20 different instances of this model, and for each instance, the Algorithm 1 converges to a Nash equilibrium. The average time to compute a Nash equilibrium is 805.31 seconds. The Figure 2 represents the variation in the time for different instances. 


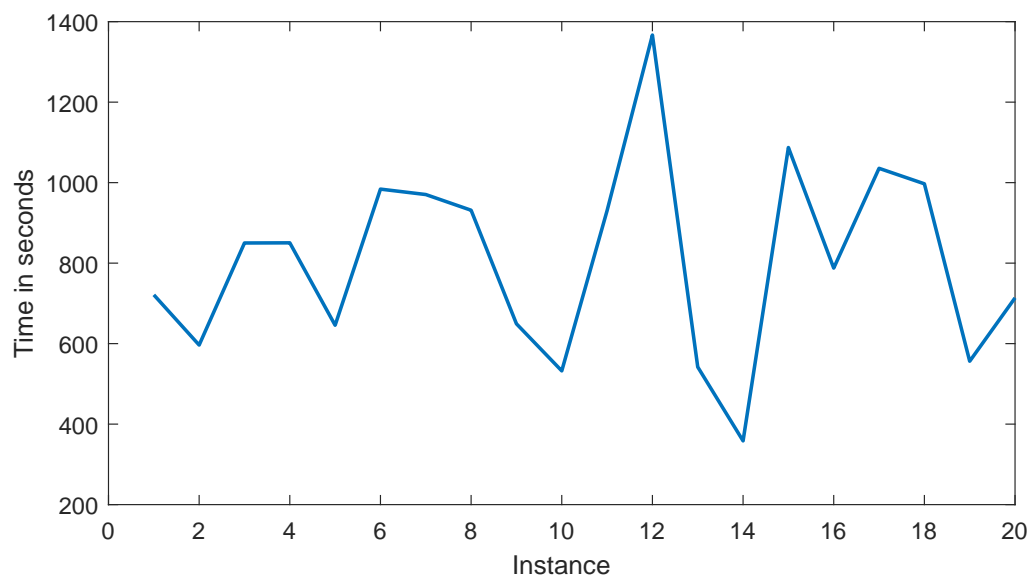

Fig. 2: Time vs Instances

\section{Conclusion}

We consider an $n$-player non-cooperative game under joint chance constraints. The random constraint vectors are independent. We identify a list of prominent probability distributions from the class of elliptical distributions. We consider the case when the probability distribution of each random constraint vector is among the identified elliptical distributions as well as the case when it is a finite mixture of the identified elliptical distributions. We derive the bounds for the players' confidence levels and the weights used in the mixture distribution and propose a new convex reformulation of the joint chance constraint when the players' confidence levels and the weights used in the mixture distributions are within the bounds. Under mild conditions, we show that there exists a Nash equilibrium of the CCG. As an application of these games, we study a competition between two investment firms from the same market. The numerical experiments are performed on randomly generated instances of different sizes by using a best response algorithm.

\section{Acknowledgement}

This research was supported by DST/CEFIPRA Project No. IFC/4117/DSTCNRS-5th call/2017-18/2 and CNRS Project No. AR/SB:2018-07-440.

\section{Appendix A Proof of Lemma 2}

Proof Let $h_{1}:[0,1] \rightarrow\left[\bar{\alpha}_{k, i}, 1\right]$ such that $h_{1}\left(z_{k}^{i}\right)=\alpha_{i}^{z_{k}^{i}}$, and $h_{2}:\left[\bar{\alpha}_{k, i}, 1\right] \rightarrow \mathbb{R}$ such that $h_{2}(p)=\log \left(\Psi_{k}^{i}\right)^{-1}(p)$ be two functions. Then, the function composition $\left(h_{2} \circ h_{1}\right)\left(z_{k}^{i}\right)=$ 
$\log \left(\Psi_{k}^{i}\right)^{-1}\left(\alpha_{i}^{z_{k}^{i}}\right)$. It is easy to see that $h_{1}\left(z_{k}^{i}\right)$ is a convex function of $z_{k}^{i}$ and $h_{2}(p)$ is a nondecreasing function. Then, from Lemma 3.11 of [24], it suffices to show that $h_{2}(p)$ is a convex function of $p$ in order to show $\log \left(\Psi_{k}^{i}\right)^{-1}\left(\alpha_{i}^{z_{k}^{i}}\right)$ is a convex function. The second order derivative of $h_{2}(p)$ is given by

$$
-\frac{u g_{k}^{\prime i}(u)+g_{k}^{i}(u)}{c^{2} u^{2}\left(g_{k}^{i}(u)\right)^{3}}
$$

where $u=\left(\Psi_{k}^{i}\right)^{-1}(p), g_{k}^{i}(u)$ is the radial density function corresponding to distribution function $\Psi_{k}^{i}(u), g_{k}^{i}(u)$ is the derivative of $g_{k}^{i}(u)$. Therefore, the function $h_{2}(p)$ is convex if

$$
\left(\Psi_{k}^{i}\right)^{-1}(p) g_{k}^{\prime i}\left(\left(\Psi_{k}^{i}\right)^{-1}(p)\right)+g_{k}^{i}\left(\left(\Psi_{k}^{i}\right)^{-1}(p)\right) \leq 0 .
$$

The verification of 20 for each distribution listed in Table 1 is as follows:

Normal Distribution: Using the radial density function of normal distribution given in Table 1 the condition 20 can be written as

$$
e^{-\frac{u^{2}}{2}}(1-u)(1+u) \leq 0
$$

where $u=\left(\Psi_{k}^{i}\right)^{-1}(p)$. From Table 1 the value of $\bar{\alpha}_{k, i}$ associated with normal distribution is $\Psi_{k}^{i}(1)$ Since, the function $g_{2}(p)$ is defined on $\left[\bar{\alpha}_{k, i}, 1\right], p \geq \bar{\alpha}_{k, i}=\Psi_{k}^{i}(1)$. Hence, the condition 21 is satisfied.

$t$ distribution: Using the radial density function of $t$ distribution given in Table 1 the condition 20 can be written as

$$
\left(1+\frac{u^{2}}{\nu}\right)^{-\frac{1+\nu}{2}-1}(1+u)(1-u) \leq 0
$$

where $u=\left(\Psi_{k}^{i}\right)^{-1}(p)$. From Table 1 the value of $\bar{\alpha}_{k, i}$ associated with $t$ distribution is $\Psi_{k}^{i}(1)$. From the similar arguments used in case of normal distribution, we have $p \geq \Psi_{k}^{i}(1)$. Hence, the condition 22 is satisfied.

Cauchy distribution: The Cauchy distribution is a special case of $t$ distribution when $\nu=1$. Therefore, the condition 20 holds in this case using the similar arguments used in case of $t$ distribution.

Laplace distribution: Using the radial density function of Laplace distribution given in Table 1 the condition 20 can be written as

$$
e^{-u}(1-u) \leq 0
$$

where $u=\left(\Psi_{k}^{i}\right)^{-1}(p)$. From Table 1 the value of $\bar{\alpha}_{k, i}$ associated with Laplace distribution is $\Psi_{k}^{i}(1)$ which gives $p \geq \Psi_{k}^{i}(1)$. Hence, the condition 23 is satisfied.

Kotz type distribution: Using the radial density function of Kotz type distribution given in Table 1 the condition 20 can be written as

$$
u^{2(N-1)} e^{-r u^{2 s}}\left(2 N-1-2 r s u^{2 s}\right) \leq 0,
$$

where $u=\left(\Psi_{k}^{i}\right)^{-1}(p)$. From Table 1 the value of $\bar{\alpha}_{k, i}$ associated with Kotz type distribution is $\Psi_{k}^{i}\left(\left(\frac{2 N-1}{2 s r}\right)^{\frac{1}{2 s}}\right)$ which gives $p \geq \Psi_{k}^{i}\left(\left(\frac{2 N-1}{2 s r}\right)^{\frac{1}{2 s}}\right)$. Hence, the condition 
(24) is satisfied.

Pearson type VII distribution: Using the radial density function of Pearson Type VII distribution given in Table 1 , the condition 20 can be written as

$$
\left(1+\frac{u^{2}}{m}\right)^{-N-1}\left(1-\frac{2 N-1}{m} u^{2}\right) \leq 0
$$

where $u=\left(\Psi_{k}^{i}\right)^{-1}(p)$. From Table 1 the value of $\bar{\alpha}_{k, i}$ associated with Pearson type VII distribution is $\Psi_{k}^{i}\left(\sqrt{\frac{m}{2 N-1}}\right)$ which gives $p \geq \Psi_{k}^{i}\left(\sqrt{\frac{m}{2 N-1}}\right)$. Hence, the condition 25 is satisfied.

\section{Appendix B Proof of Lemma 5}

Proof The second order derivative of $\Psi_{k, m}^{i}\left(e^{\tau_{k, m}^{i}}\right)$ is

$$
\Psi_{k, m}^{\prime \prime} i\left(e^{\tau_{k, m}^{i}}\right)\left(e^{2 \tau_{k, m}^{i}}\right)+\Psi_{k, m}^{\prime i}\left(e^{\tau_{k, m}^{i}}\right)\left(e^{\tau_{k, m}^{i}}\right),
$$

where $\Psi_{k, m}^{\prime i}(\cdot)$ and $\Psi_{k, m}^{\prime \prime}(\cdot)$ are the first and second order derivative functions of $\Psi_{k, m}^{i}(\cdot)$, respectively. Let $g_{k, m}^{i}(\cdot)$ be a radial density associated with the distribution function $\Psi_{k, m}^{i}(\cdot)$. Then, the concavity of $\Psi_{k, m}^{i}\left(e^{\tau_{k, m}^{i}}\right)$ is equivalent to

$$
g_{k, m}^{\prime i}\left(e^{\tau_{k, m}^{i}}\right)\left(e^{\tau_{k, m}^{i}}\right)+g_{k, m}^{i}\left(e^{\tau_{k, m}^{i}}\right) \leq 0
$$

The verification of 26 for each distribution listed in Table 2 is as follows:

Normal distribution: The radial density function of normal distribution is $g_{k, m}^{i}(u)=$ $e^{-\frac{u^{2}}{2}}$ and its first derivative is $g_{k, m}^{\prime i}(u)=-u e^{-\frac{u^{2}}{2}}$. The condition 26 can be written as

$$
e^{-\frac{u^{2}}{2}}(1-u)(1+u) \leq 0
$$

where $u=e^{\tau_{k, m}^{i}}$. From Table 2 the weight $w_{k, m}^{i}$ associated with normal distribution is such that $w_{k, m}^{i} \geq \frac{1}{1-\Psi_{k, m}^{i}(1)}\left(1-\alpha_{i}\right)$. Then, from Lemma $4 . r_{k, m}^{i} \geq 1$ which in turn implies that $e^{\tau_{k, m}^{i}} \geq 1$. Hence, the condition 27 is satisfied.

$t$ distribution: Using the radial density function of $t$ distribution given in Table 1 the condition 26) can be written as

$$
\left(1+\frac{u^{2}}{\nu}\right)^{-\frac{1+\nu}{2}-1}(1+u)(1-u) \leq 0
$$

where $u=e^{\tau_{k, m}^{i}}$. As similar to the case of normal distribution, the weight $w_{k, m}^{i}$ associated with $t$ distribution is such that $w_{k, m}^{i} \geq \frac{1}{1-\Psi_{k, m}^{i}(1)}\left(1-\alpha_{i}\right)$. From the similar arguments used in the case of normal distribution, the condition 28 is satisfied.

Cauchy distribution: The Cauchy distribution is a special case of $t$ distribution when $\nu=1$. Therefore, the condition 26. holds in this case using the similar arguments used in the case of $t$ distribution.

Laplace distribution: Using the radial density function of Laplace distribution given in Table 1 the condition 26 can be written as

$$
e^{-u}(1-u) \leq 0,
$$


where $u=e^{\tau_{k, m}^{i}}$. From Table 2 the weight $w_{k, m}^{i}$ associated with Laplace distribution is such that $w_{k, m}^{i} \geq \frac{1}{1-\Psi_{k, m}^{i}(1)}\left(1-\alpha_{i}\right)$. Then, from Lemma $4 r_{k, m}^{i} \geq 1$ which in turn implies that $e^{\tau_{k, m}^{i}} \geq 1$. Hence, the condition 29 is satisfied.

Kotz type distribution: Using the radial density function of Kotz type distribution given in Table 1 the condition 26] can be written as

$$
u^{2(N-1)} e^{-r u^{2 s}}\left(2 N-1-2 r s u^{2 s}\right) \leq 0
$$

where $u=e^{\tau_{k, m}^{i}}$. From Table 2 the weight $w_{k, m}^{i}$ associated with Kotz type distribution is such that $w_{k, m}^{i} \geq \frac{1}{1-\Psi_{k, m}^{i}\left(\frac{2 N-1}{2 s r}\right)^{\frac{1}{2 s}}}\left(1-\alpha_{i}\right)$. Then, from Lemma $44 r_{k, m}^{i} \geq\left(\frac{2 N-1}{2 s r}\right)^{\frac{1}{2 s}}$ which in turn implies that $e^{\tau_{k, m}^{i}} \geq\left(\frac{2 N-1}{2 s r}\right)^{\frac{1}{2 s}}$. Hence, the condition 30 is satisfied.

Pearson Type VII distribution: Using the radial density function of Pearson Type VII distribution given in Table 1 the condition 26 can be written as

$$
\left(1+\frac{u^{2}}{m}\right)^{-N-1}\left(1-\frac{2 N-1}{m} u^{2}\right) \leq 0
$$

where $u=e^{\tau_{k, m}^{i}}$. From Table 2 the weight $w_{k, m}^{i}$ associated with Pearson Type VII is such that $w_{k, m}^{i} \geq \frac{1}{1-\Psi_{k, m}^{i}\left(\sqrt{\frac{m}{2 N-1}}\right)}\left(1-\alpha_{i}\right)$. Then, from Lemma $4 r_{k, m}^{i} \geq \sqrt{\frac{m}{2 N-1}}$ which in turn implies that $e^{\tau_{k, m}^{i}} \geq \sqrt{\frac{m}{2 N-1}}$. Hence, the condition 31 is satisfied.

\section{References}

1. Basar, T., Olsder, G.J.: Dynamic noncooperative game theory, 2nd edn. SIAM, Philadelphia, PA (1999)

2. Blau, R.A.: Random-payoff two person zero-sum games. Operations Research 22(6), 1243-1251 (1974)

3. Buckley, I., Saunders, D., Seco, L.: Portfolio optimization when asset returns have the Gaussian mixture distribution. European Journal of Operational Research 185, 1434-1461 (2008)

4. Cassidy, R.G., Field, C.A., Kirby, M.J.L.: Solution of a satisficing model for random payoff games. Management Science 19(3), 266-271 (1972)

5. Charnes, A., Kirby, M.J.L., Raike, W.M.: Zero-zero chance-constrained games. Theory of Probability and its Applications 13(4), 628-646 (1968)

6. Cheng, J., Leung, J., Lisser, A.: Random-payoff two-person zero-sum game with joint chance constraints. European Journal of Operational Research 251(1), 213-219 (2016)

7. Cheng, J., Lisser, A.: A second-order cone programming approach for linear programs with joint probabilistic constraints. Operations Research Letters 40(5), 325-328 (2012)

8. Conejo, A.J., Nogales, F.J., Arroyo, J.M., García-Bertrand, R.: Riskconstrained self-scheduling of a thermal power producer. IEEE Transactions on Power Systems 19(3), 1569-1574 (2004) 
9. Couchman, P., Kouvaritakis, B., Cannon, M., Prashad, F.: Gaming strategy for electric power with random demand. IEEE Transactions on Power Systems 20(3), 1283-1292 (2005)

10. Debreu, G.: A social equilibrium existence theorem. Proceedings of National Academy of Sciences 38, 886-893 (1952)

11. Fan, K.: Applications of a theorem concerning sets with convex sections. Mathematische Annalen 163, 189-203 (1966)

12. Fang, K.T., Kotz, S., Ng, K.W.: Symmetric Multivariate and Related Distributions. Chapman and Hall, London, New York (1990)

13. Giacomini, R., Gottschling, A., Haefke, C., White, H.: Mixtures of tdistributions for finance and forecasting. Journal of Econometrics 144 175-192 (2008)

14. Henrion, R.: Structural properties of linear probabilistic constraints. Optimization 56(4), 425-440 (2007)

15. Henrion, R., Strugarek, C.: Convexity of chance constraints with independent random variables. Computational Optimization and Applications 41(2), 263-276 (2008)

16. Kamdem, J.S.: Value-at-risk and expected shortfall for linear portfolios with elliptically distributed risk factors. International Journal of Theoretical and Applied Finance 8, 537-551 (2005)

17. Kamdem, J.S.: $\delta$-VaR and $\delta$-TVaR for portfolios with mixture of elliptic distributions risk factors and dcc. Insurance: Mathematics and Economics 44, 325-336 (2009)

18. Kannan, A., Shanbhag, U.V., Kim, H.M.: Addressing supply-side risk in uncertain power markets: stochastic Nash models, scalable algorithms and error analysis. Optimization Methods and Software 28(5), 1095-1138 (2013)

19. Kociski, M.: Transaction costs and market impact in investment management. e-Finanse: Financial Internet Quarterly 10, 28-35 (2014)

20. Liu, J., Lisser, A., Chen, Z.: Stochastic geometric optimization with joint probabilistic constraints. Operations Research Letters 44, 687-691 (2016)

21. Lobo, M.S., Fazel, M., Boyd, S.: Portfolio optimization with linear and fixed transaction costs. Annals of Operations Research 152, 341-365 (2007)

22. Mazadi, M., Rosehart, W.D., Zareipour, H., Malik, O.P., Oloomi, M.: Impact of wind integration on electricity markets: A chance-constrained Nash Cournot model. International Transactions on Electrical Energy Systems 23(1), 83-96 (2013)

23. Nash, J.F.: Equilibrium points in n-person games. Proceedings of the National Academy of Sciences 36(1), 48-49 (1950)

24. Peng, S., Singh, V.V., Lisser, A.: General sum games with joint chance constraints. Operations Research Letters 56, 482-486 (2018)

25. Ravat, U., Shanbhag, U.V.: On the characterization of solution sets of smooth and nonsmooth convex stochastic Nash games. SIAM Journal of Optimization 21(3), 1168-1199 (2011) 
26. Singh, V.V., Jouini, O., Lisser, A.: Existence of Nash equilibrium for chance-constrained games. Operations Research Letters 44(5), 640-644 (2016)

27. Singh, V.V., Lisser, A.: A characterization of Nash equilibrium for the games with random payoffs. Journal of Optimization Theory and Applications 178(3), 998-1013 (2018)

28. Singh, V.V., Lisser, A.: A second order cone programming formulation for zero sum game with chance constraints. European Journal of Operational Research 275, 839-845 (2019) 